Georg M. Bongartz

Received: 21 March 2003

Accepted: 28 March 2003

Published online: 29 April 2003

(C) Springer-Verlag 2003
G. M. Bongartz ( $)$

Department of Diagnostic Radiology, University Hospitals Basel, Kantonsspital, Petersgraben 4, 4031 Basel, Switzerland e-mail: gbongartz@uhbs.ch Tel.: +41-61-2654344

Fax: +41-61-2654354

\title{
Contrast media in magnetic resonance angiography
}

In the past years, magnetic resonance angiography (MRA) has been recognised as a powerful tool in the evaluation of vascular disease in a majority of anatomic territories. It may be applied with or without contrast media depending on the area of investigation, on the physiological properties of the interesting vessels, and mainly on the dedicated clinical question [1].

Native techniques are still advocated for the evaluation of the intracranial circulation and in some places for the detection of carotid artery disease as well as for detection of peripheral arterial pathologies, because in these anatomical areas motion of the vessels is limited to minor pulsation. This allows for longer imaging periods, correlated with beneficial higher resolution of the resulting images. In other vascular territories, vessels are affected by secondary motion such as breathing or peristalsis, and related artefacts prevent the application of longer-lasting MRA measurements. Other inherent problems of native techniques, such as saturation effects in time-of-flight (TOF) MRA and phase wrapping in phase-contrast (PC) MRA, are well known and further support the introduction of contrast-media-enhanced vascular studies.

In contrast-enhanced (CE) MRA, the arterial first pass of an intravenously applied bolus of contrast media must be coordinated with the data acquisition. Meanwhile, this has successfully been applied to all major arterial pathologies and the technique is under permanent evolution. Various studies on the direct comparison of CE MRA to intra-arterial digital subtraction angiography (DSA) demonstrate the high potential of the lesser-invasive MR technique and its advantage concerning the three-dimensional approach to the vessel and the pathology. In contrast, insufficiencies in in-plane resolution and post-processing artefacts in 3D CE MRA have also been described. The stability of the bolus during the most sensitive part of the data acquisition period, namely at the acquisition of the central lines in $\mathrm{k}$-space, depends on a variety of factors [2,3]; among these, the distribution of the CM bolus, the CM relaxivity, and the local flow physiology are the most important. In a routine clinical setting, the investigator adjusts injection rate, timing and amount of contrast media to the measurement sequence in order to achieve maximum shortening of $\mathrm{T} 1$ values explicitly within the investigated vasculature [4]. Especially for the diagnostic workup of peripheral arterial occlusive disease of the lower extremities this problem increases due to the length of the interesting field of view. Several measurements have to be added and the bolus geometry must be adjusted to the entire diagnostic area $[3,4]$. 
Optimised conditions for the visualisation of the lower limb arteries could be provided by a stable and long-lasting CM supply as by bloodpool agents, because this enables longer measuring times and thus high resolution. But so far the enhancement of the superimposing veins prevents the clinical use as equilibrium CM.

Venous signal contamination of the arterial display in first-pass MRA not only occurs in normal subjects but to a much stronger extent in arteriosclerotic patients. It is therefore one of the most problematic handicaps for peripheral MRA. Faster data acquisition for each of the investigated volumes is recommended at the price of resolution or signal-to-noise ratio (SNR). In order to compensate for decreased SNR, CM with stronger relaxivity effects can be applied.

Various new contrast agents for MRA are recently under development driven by the clinical demand for more intensive, more specific or longer-lasting enhancement characteristics. Most of these contrast agents are based on gadolinium compounds chelated to complicated molecules. Two papers published in this issue of "European Radiology" present the results of multicentric trials on a new $1.0 \mathrm{M} \mathrm{Gd}$ preparation $[5,6]$. While the safety issues of the new compound are thoroughly tested in one study without regard to the clinical value of the investigation, the second paper focuses on the implications of the new CM on peripheral MRA.

With its large safety profile, the tested CM presents many similarities to one of the most often applied agents: $0.5 \mathrm{M}$ Gd-DTPA which has been in use for approximately 20 years with only a low rate of complications [7]. In the multicentric study presented here [5], the CM was tested not only for possible side effects but the drug also underwent a more detailed safety evaluation than in most comparable phase-III Gd trials. Therein, effects on the renal function were under special focus. The detailed analysis of the excretion mechanism and the nephrologi- cal effects demonstrated a broad renal tolerance [8]. Moreover, the stability of the Gd compound as defined by the low transmetallation effect on serum zinc values was investigated.

The properties of gadobutrol $1 \mathrm{M}$ for peripheral CE MRA were demonstrated with direct comparison with intra-arterial DSA in the second paper [6]. The aim of that study was to identify the equity of either method for the diagnostic of peripheral arterial occlusive disease. The options of an improved bolus geometry for sufficient and selective arterial contrast effect in the peripheral circulation have previously been discussed and were recommended for 3D MRA [9].

Recent studies have addressed the benefits of the higher-concentrated Gd chelate by direct comparison with a standard concentration of $0.5 \mathrm{M}$ in volunteers [10]. Future prospective trials on patients with peripheral arteriosclerotic disease are required to demonstrate the positive effects in a clinical setting.

\section{References}

1. Bongartz GM, Boos M, Winter K, Ott H, Scheffler K, Steinbrich W (1997) Clinical utility of contrast-enhanced MR angiography. Eur Radiol 7 (Suppl 5):178-186

2. Lee JJ, Chang Y, Tirman PJ, Ryum HK, Lee SK, Kim YS, Kang DS (2001) Optimizing of gadolinium-enhanced MR angiography by manipulation of acquisition and scan delay times. Eur Radiol 11:754-766

3. Boos M, Lentschig M, Scheffler K, Bongartz GM, Steinbrich W (1998) Contrast-enhanced magnetic resonance angiography of peripheral vessels. Different contrast agent applications and sequence strategies: a review. Invest Radiol 33:538-546

4. Carroll TJ, Korosec FR, Swan JS, Hany TF, Grist TM, Mistretta CA (2001) The effect of injection rate on time-resolved contrast-enhanced peripheral MRA. J Magn Reson Imaging 14:401-410
5. Balzer JO, Loewe C, Davis K, Goyen M, Leiner T, Meaney J, PöcklerSchöniger C, Schulte-Altedorneburg G, Tombach B, Vosshenrich R, Wegener R (2003) Safety of contrast-enhanced MR-angiography employing gadobutrol 1.0 M as contrast material: experience in 436 patients. Eur Radiol DOI 10.1007/s00330-002-1768-5

6. Hentsch A, Aschauer MA, Balzer JO, Brossmann J, Busch HP, Davis K, Douek P, Ebner F, van Engelshoven JMA, Gregor M, Kersting C, Knüsel PR, Leen E, Leiner T, Loewe C, McPherson S, Reimer P, Schäfer FKW, Taupitz M, Thurnherr SA, Tombach B, Wegener R, Weishaupt D, Meaney J (2003) Gadobutrol-enhanced movingtable magnetic resonance angiography in patients with peripheral vascular disease: a prospective, multi-centre blinded comparison with digital subtraction angiography. Eur Radiol DOI 10.1007/s00330-003-1844-5

7. Niendorf HP, Dinger JC, Haustein J, Cornelius I, Alhassan A, Clauss W (1991) Tolerance data of Gd-DTPA: a review. Eur J Radiol 13:15-20
8. Tombach B, Bremer C, Reimer P, Kisters K, Schaefer RM, Geens V, Heindel W (2001) Renal tolerance of a neutral gadolinium chelate (gadobutrol) in patients with chronic renal failure: results of a randomized study. Radiology 218:651-657

9. Adam G, Neuerburg J, Spuntrup E, Muhler A, Scherer K, Gunther RW (1994) Dynamic contrast-enhanced MR imaging of the upper abdomen: enhancement properties of gadobutrol, gadolinium-DTPA-polylysine, and gadolinium-DTPA-cascade-polymer. Magn Reson Med 32:622-628

10. Goyen M, Lauenstein TC, Herborn CU, Debatin JF, Bosk S, Ruehm SG (2001) 0.5 M Gd chelate (Magnevist) versus 1.0 M Gd chelate (Gadovist): dose-independent effect on image quality of pelvic three-dimensional MR-angiography. J Magn Reson Imaging 14:602-607 